\title{
A novel system for measuring optical properties in arterial blood of man
}

doi:10.1533/abbi.2005.0032

\author{
A. Castañeda-Miranda and L. D. L. Vizcaya \\ Engineering E Industrial Development Center (CIDESI), Santiago de Queretaro, Queretaro, Mexico
}

\begin{abstract}
The necessity of man-machine communication has been increasing day by day because of the great number of approaches that help to make human life agile or comfortable. Health is one of the aspects that has generated more interest in recent years. The optical technologies are new technical tools that are being implemented for medical diagnosis and for describing various processes occurring within human blood. These techniques have the virtue of being nondestructive; in addition they have the advantage of remote direction by using an in situ technique, with sensitivity capable of studying the properties of the blood through electromagnetic excitation. A device to obtain the electromagnetic spectrum in the blood has being designed and is presented in this work. This device uses a quartz lamp, emitting electromagnetic radiation between $200 \mathrm{~nm}$ and $2200 \mathrm{~nm}$. A model for measuring the light attenuation through the blood is used. By applying a control in the frequency domain, a circuit device is designed. This device uses a database in time domain for its subsequent analysis by Fast Fourier Transformation in order to obtain absorption spectra. The acquisition time is of the order of microseconds, and the system is controlled automatically through accessible software from a personal computer.
\end{abstract}

Keywords: Biomechanics, medical instrumentation, properties of blood, bioelectronics.

\section{INTRODUCTION}

Feedback systems are found not only in the field of engineering but also in various fields detached from it. For example, the human body is a very advanced feedback control system. The corporal temperature, as well as the sanguine pressure, is maintained constant through a physiological pressure. In fact, the feedback accomplishes a vital function: it allows human beings, which are relatively insensitive to external disturbances, to adapt to changing environments. In order to achieve this feedback, the blood acts as a distribution system for various cell concentrations. The system described here allows measurement of some blood properties through the application of an optical test, one which, concurrently, has the characteristics of both being noninvasive and being in situ; it also has a complex configuration and involves coupling of all the parts. Similarly, an optical system based

Corresponding Author:

A. Castañeda-Miranda

Engineering \& Industrial Development Center (CIDESI)

Av. Playa Pie de la Cuesta No. 702. Desarrollo San Pablo

Santiago de Queretaro, Queretaro, Mexico

Tel: +52-442-2119800x404; Fax: +52-442-2119839

Email: acastaneda@cidesi.mx on the blood optical properties is developed. The guiding principle of this study is based on the fact that indirect knowledge of the intensity of a luminous beam, as well as of the changes that it makes on time and space, can assist in building detection or alteration systems (using, e.g. a commercial lamp) to determine the curvature radius, superficial roughness, internal angles and other parameters of the surface under test (Jacques 1993; Cysewska-Sobuslack 1994; Javidi and Horner 1994; Nitzan et al. 1996; Gotshal et al. 1997; Zhbankov et al. 2000), the radiation detector being an essential part (Gotshal et al. 1997; Zhbankov et al. 2000). Besides the interaction of the electromagnetic radiation with the tissue structure, a great variety of phenomena can be produced taking advantage of the different technological manufacturing devices. In this device, it is possible to detect various processes through optical transitions that can be collected through the combination of the optics, computer programs, electronics and mechanics. Therefore, the margin of application and operation is based on the properties of the radiation of the diode detectors. These are entrusted with the conversion of an optical signal into an electrical signal (Gotshal et al. 1997). The physical detection of light interaction consists of a diode of indirect band gap that acts as a photodetector when the $\mathrm{P}-\mathrm{N}$ junction or diode is illuminated through a window with sufficient radiation. This process transmits the necessary energy for excitation of electrons from the valence 


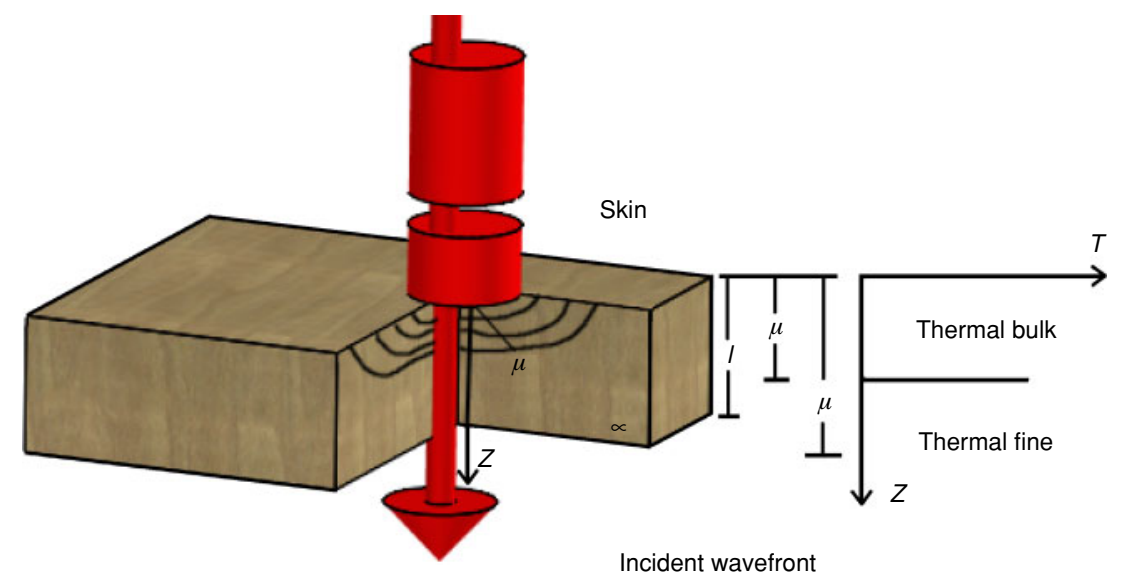

Figure 1 Behavioral illustrations of fine and bulk thermal materials.

band to the conduction band, resulting in a current of free electrons in the form of light photons (photocurrent). The photodiode is operated by connecting it to a charge resistance, thus transforming the photocurrent to a voltage difference. A typical sensitivity of these devices is of the order of microamperes for a microwatt of incident light, which as a rule, is affected by far parasite signals independent of the noise of the luminous interest signal (white noise). Then, taking advantage of the properties of an operational amplifier (AOP) for amplifying the signal amplitude originated at the photodiode, a low pass filter is designed for the output treatment of the device, which allows passage of only low frequencies that contain the signal of interest. This configuration is integrative, and consists of a resistance as well as a capacitor, both connected in a parallel manner between the extremities of the output and the inverse terminal of the AOP as shown in Figure 2. One of the important applications of these devices is the Fourier Transformed Infrared Spectroscopy, the one that helps in the study of the absorption of the electromagnetic radiation by the matter. This is obtained in the same manner as atomic and molecular spectra, providing detailed information about the structure (symmetry, distance and angles between liaison) in addition to the properties of the material (electronic distribution, liaison force and intermolecular process) (Cysewska-Sobuslack 1994).

\section{OPTICS AND THERMAL PROPERTIES OF THE BLOOD}

Notations for the material properties for the optical or thermal diffusion mechanism are shown in Table 1. The aim of the proposed model is to determine these properties.

From the optical viewpoint, materials can be classified according to their optical opaqueness, which is determined by the relationship $\mu=1 / a_{i}$, where $\mu$ is the optical absorption length and $a$ is the thickness of the sample $i$. Thus, according to the optical opaqueness, the materials can be divided in two main groups: (a) Optically transparent materials: A material is optically transparent when all of the light intensity that incidences on the surface of a sample is absorbed throughout the thickness of the sample. This is expressed mathematically as $\mu_{\mathrm{s}}>1$.

(b) Optically opaque materials: In this case, the light intensity that incidences on the surface of the material is attenuated thoroughly much before crossing the sample. It is given as $\mu_{\mathrm{s}} \gg 1$.

On the other hand, from the thermal viewpoint, a material can be categorised according to the relationship between the magnitude of the thermal diffusion length, defined as $\mu_{\mathrm{s}}$, and the thickness of the sample:

$$
\mu_{\mathrm{s}}=\sqrt{\frac{\alpha}{\pi f}}
$$

(a) Thermal fine materials: A thermal material is considered when the thermal diffusion length $\mu_{s}$ is greater than the thickness of the sample (i.e. $\mu_{\mathrm{s}}>1$ ), as can be seen in Figure 1.

(b) Thermal bulk materials: These are characterised by the fact that the thermal wave is attenuated in the interior of the sample (i.e. $\left.\mu_{\mathrm{s}}<1\right)$.

In this work, we consider that all thermal bulk materials are optically opaque. In Figure 1, the thermal behavior described above can be observed.

\section{FREQUENCY DOMAIN MODEL FOR THE PHOTODIODE}

In the model, splitting of the photodiode occurs into a photocurrent source, a capacitor as the junction capacitance of the diode $\left(C_{\mathrm{D}}\right)$ and a resistance $\left(R_{\mathrm{D}}\right)$, all of them connected in a parallel manner (see Figure 2). Later the photodiode is connected to an AOP, thus permitting an electrical analysis based on the Kirchhoff's Law. In this way, the total impedance of the circuit and therefore a frequency behavior model is obtained through a transfer function (Bode chart). 
Table 1 Notation for the description of optical signals

\begin{tabular}{lll}
\hline Parameter & Denomination & Unit \\
\hline$K_{j}$ & Thermal conductivity of material $j$ & $\mathrm{~W} / \mathrm{cm} \mathrm{K}$ \\
$\rho_{j}$ & Density of material $j$ & $\mathrm{~g} / \mathrm{cm}^{3}$ \\
$C_{j}$ & Specific heat of material $j$ & $\mathrm{~W} / \mathrm{g} \mathrm{K}$ \\
$\omega$ & Modulation frequency of beam & $\mathrm{rad} / \mathrm{s}$ \\
$\alpha_{j}$ & Thermal diffusion of material $j$ & $\mathrm{~cm}^{2} / \mathrm{s}$ \\
$a_{j}$ & Diffusion thermal coefficient of material $j$ & $\mathrm{~cm}^{-1}$ \\
$\sigma_{j}$ & Complex coefficient of thermal diffusion & $\mathrm{cm}^{-1}$ \\
$\mu_{j}=1 / a_{j}$ & Thermal diffusion length of material $j$ & $\mathrm{~cm}$ \\
$l_{\mathrm{s}}$ & Thickness of the sample & $\mathrm{cm}$ \\
$\beta$ & Optical absorption coefficient & $\mathrm{cm}$ \\
$\mu_{\beta}$ & Optical length of the absorption & $\mathrm{cm}$ \\
\hline
\end{tabular}

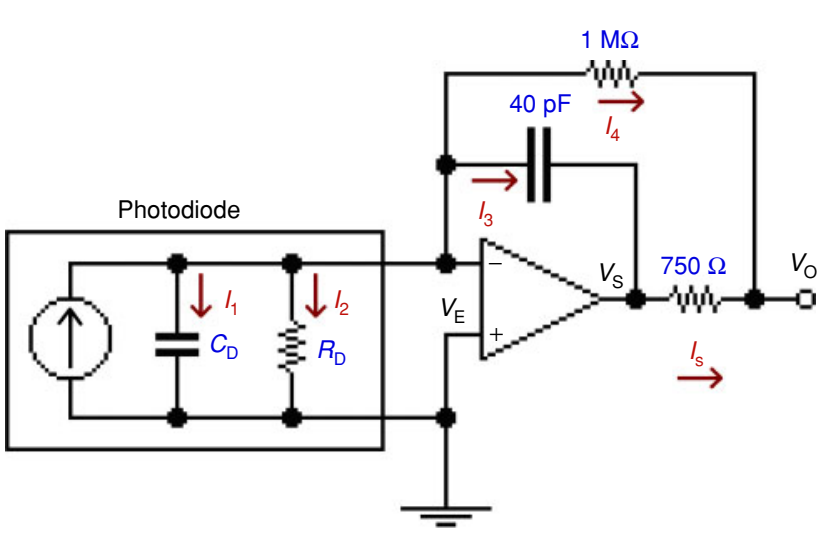

Figure 2 Model of the photodiode and operational amplifier.
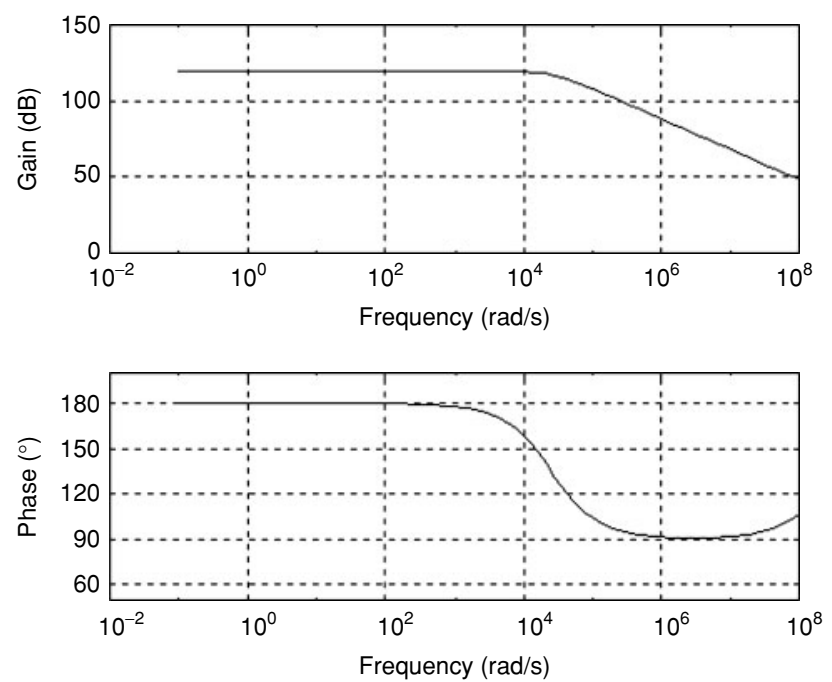

Figure 3 Graph of Bode for the photodiode model.

An analysis using Kirchhoff's Voltage Law is done according to the scheme shown in Figure 2. This scheme allows the dynamic resistance of the diode, which is very large and is the order of $\mathrm{M} \Omega\left(R_{\mathrm{D}} \gg 1\right)$. The following output function for the system is obtained and the basic equations are:

$$
I=I_{1}+I_{2}+I_{3}+I_{4}
$$

$$
\begin{aligned}
I_{1} & =s\left(V_{\mathrm{E}} C_{\mathrm{D}}\right) \\
I_{2} & =\frac{V_{\mathrm{E}}}{R_{\mathrm{D}}} \\
I_{3} & =\left(V_{\mathrm{E}}-V_{\mathrm{S}}\right) s C_{\mathrm{F}} \\
I_{4} & =\frac{V_{\mathrm{e}}-V_{0}}{R} \\
I_{\mathrm{S}} & =\frac{V_{\mathrm{S}}-V_{0}}{R_{\mathrm{S}}} \\
\Delta_{V} & =V_{0} / V_{\mathrm{E}}
\end{aligned}
$$

Combining Equations (2)-(8), we obtain the following transfer equations:

$\frac{V_{0}}{I}=$

$\frac{s\left(\Delta_{V} R R_{\mathrm{S}} C_{\mathrm{F}}-R R_{\mathrm{S}} C_{\mathrm{F}}\right)+\Delta_{V} R}{s\left(R C_{\mathrm{F}}-\Delta_{V} R C_{\mathrm{F}}+\Delta_{V} R_{\mathrm{S}} C_{\mathrm{F}}-R_{\mathrm{S}} C_{\mathrm{F}}\right)+R_{\mathrm{S}} C_{\mathrm{D}}+1-\Delta_{V}}$

If we substitute typical values for the photodiode (OPT101 of Burr-Brown), that is, capacitance $\left(C_{\mathrm{D}}=1200 \mathrm{pF}\right), \Delta V_{0}=90$ $\mathrm{dB}$ (or $\left.\Delta V_{0}=10^{5}\right)$, feedback resistance $\left(R_{\mathrm{F}}=1 \mathrm{M} \Omega\right)$, capacitor of feedback $\left(C_{\mathrm{F}}=11 \mathrm{pF}\right)$, and considering the poles and zeroes of Equation (9), we obtain the Bode plot shown in Figure 3, where the gain is given in $\mathrm{dB}$ and the phase in degrees. As it is shown in Figure 3, on having a phase change from $180^{\circ}$ to $90^{\circ}$, the cut frequency $\left(f_{\mathrm{c}}\right)$ of the filter (frequency that attenuates the signal at a rate of $20 \mathrm{~dB}$ per decade) is found. Then, to be precise, the open-loop gain has an $f_{\mathrm{c}}$ at $120^{\circ}$ of phase. This behavior of the signal is known as a low pass filter that ideally allows only low frequencies (lower than $f_{\mathrm{c}}$ ) to pass. Experimental results were obtained in order to prove the efficiency of the model. The experiment was developed as follows. A LED was placed on the active area of the photodiode and, simultaneously, the LED was connected to a signal generator using a square wave function with an amplitude between 0 and $5 \mathrm{~V}$, thus permitting variations in the frequency of the signal. On the other hand, the photodiode output was connected to a spectral analyser measuring the open-loop gain of the device 


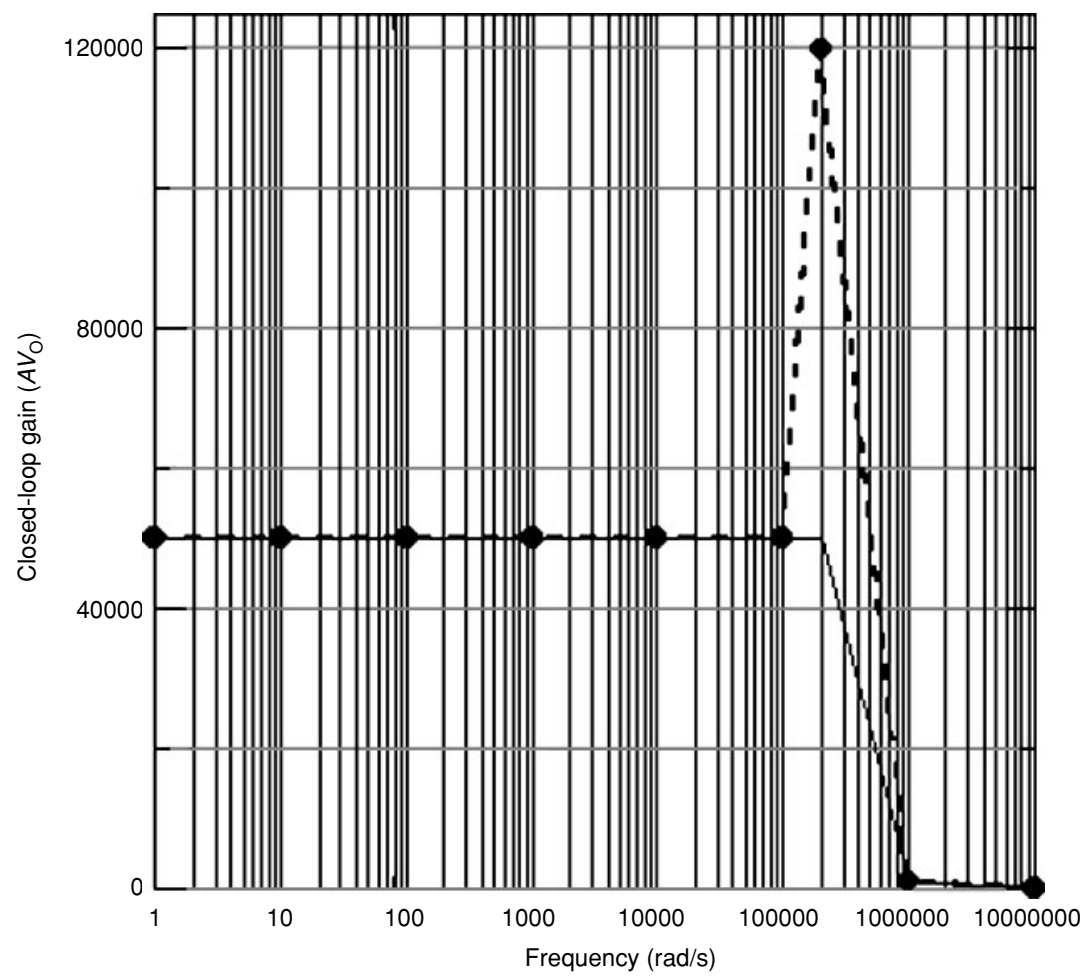

Figure 4 Experimental results of the model.

at different frequencies. The experimental results are shown in Figure 4.

Figure 4 shows a closed-loop gain plot as a function of frequency. We observe two results for the behavior of the device: one represented by the solid line, which corresponds to the theoretical result of our model, and the other, represented by the dashed line, corresponds to the experimental result. The experimental response of the device matches the predicted behavior of the theoretical model, except for some cases near the cut frequency. This is due to the presence of a resonance phenomenon. In addition, decay slope is different because more factors should be included in the analysis such as the environment temperature, dissipated temperature, and, principally, the stability of the voltage dual source that feeds the AOP.

\section{DESIGN AND CONSTRUCTION OF THE OPTOMECHANICAL DEVICE}

In order to achieve the blood parameter measurement by an optical method, a modulated system is needed that can detect the changes in amplitude and phase of the acquired signal. This is done by utilising a light detector (silicon detector) along with a white light source (lamp). The lamp generates different wavelengths from the absorption region of the spectrum after impacting normally on the finger surface. In this manner, the light beam reflected by the surface of the sample is collected by a detector, as well as the transmitted light that is absorbed by an additional detector.
The optical configuration of the experimental arrangement (see Figure 5) has a light source that impacts normally on the sample. In its path, the beam is modulated with the aid of a chopper (motor of direct current coupled to a perforated disk). Thereafter, the luminous flow is controlled through a diaphragm, which has a maximum opening of $8 \mathrm{~mm}$, then the light impacts on the finger, and finally it is collected by detectors. The detected signal corresponds to both the dispersed radiation and the radiation transmitted through the finger.

In the design of the optical system, it is necessary that the response of the lamp should be suitable to the problem as the lamp constitutes a white light source. The criterion for selecting the lamp is based on the percentage of light emission intensity with respect to the wavelength and linearity of the lineal response in a wide spectral region. According to the above-mentioned criterion, the lamps of quartz filament are less invariant with respect to the wavelength (they have less abrupt transitions throughout their spectral response). Therefore, once the lamp of suitable dimensions is obtained, the characteristics of the power supply for the lamp is taken into account. The source is designed for consuming $36 \mathrm{~W}$ of energy. This is accomplished through a $12 \mathrm{~V}$ source with a maximum current of $5 \mathrm{~A}$. The quartz lamp has a maximum intensity between $600 \mathrm{~nm}$ and $700 \mathrm{~nm}$. This radiation corresponds to the red and the near infrared regions of the electromagnetic radiation, where the rotational, longitudinal and transverse vibration modes of the CHON group for red globules are excited (Vo-Dinh 2003). 


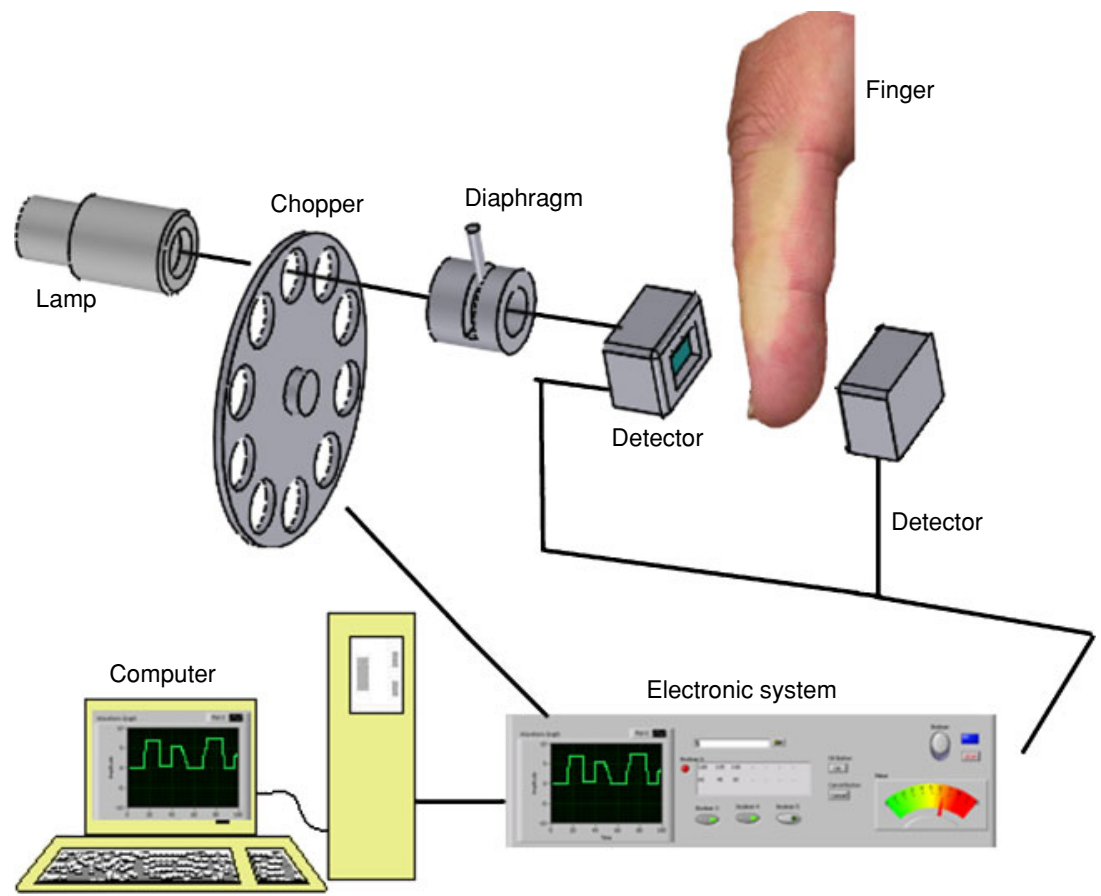

Figure 5 Diagrammatic assembly of the photoplethysmographic system.

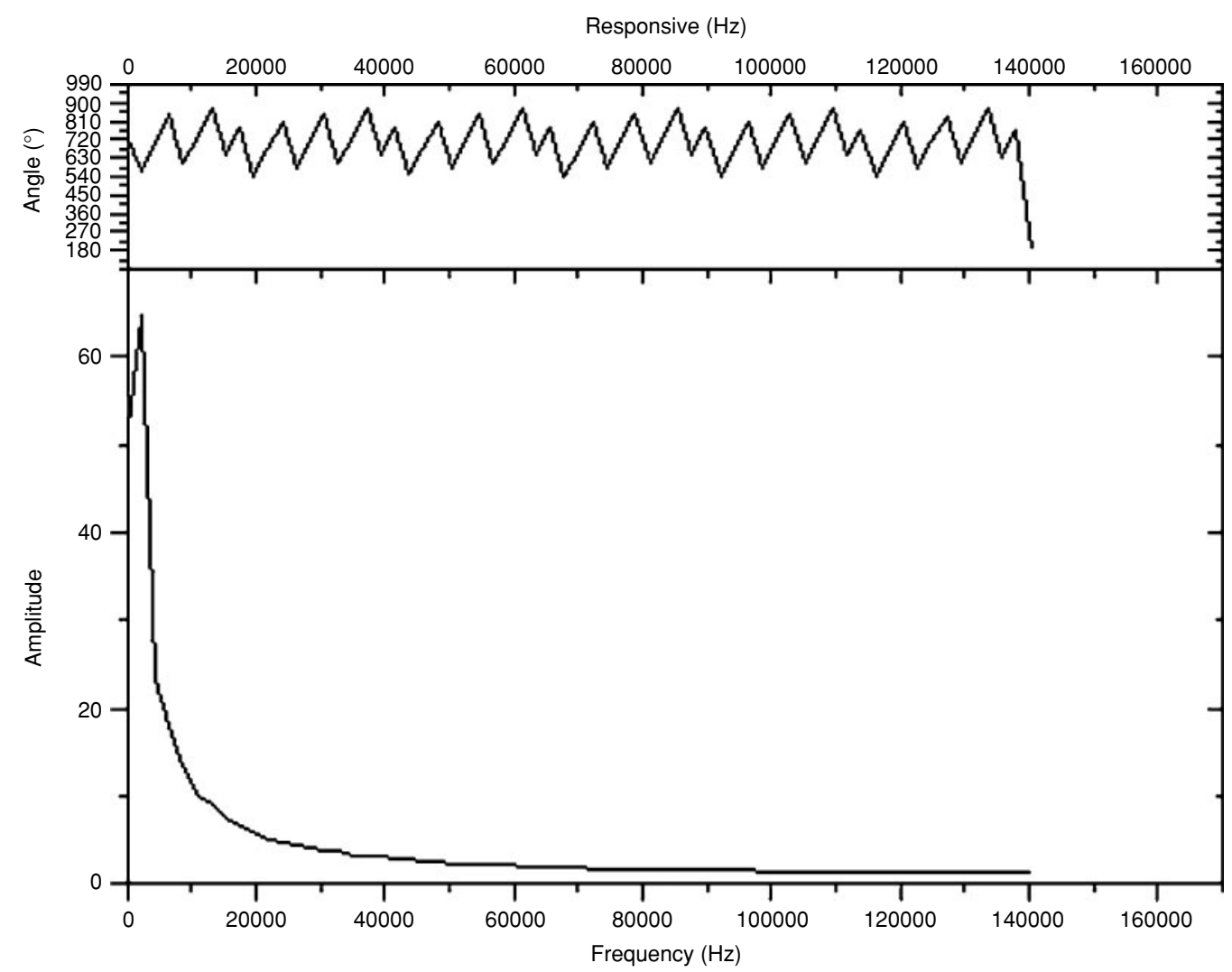

Figure 6 Response of the blood through Fast Fourier Transform analysis.

\section{RESULTS}

On obtaining a database that contains the amplitude of the optical signal as a function of time, we switch to the space frequency domain through the Fast Fourier Transform analysis (Equation (10)), taking into account that the optical signal is modulated periodically. The experimental result is shown in Figure 6. Now, using the relationship defined in Equation (11) and taking the database into account (extent amplitude versus frequency), we can give solutions for $\lambda$ 


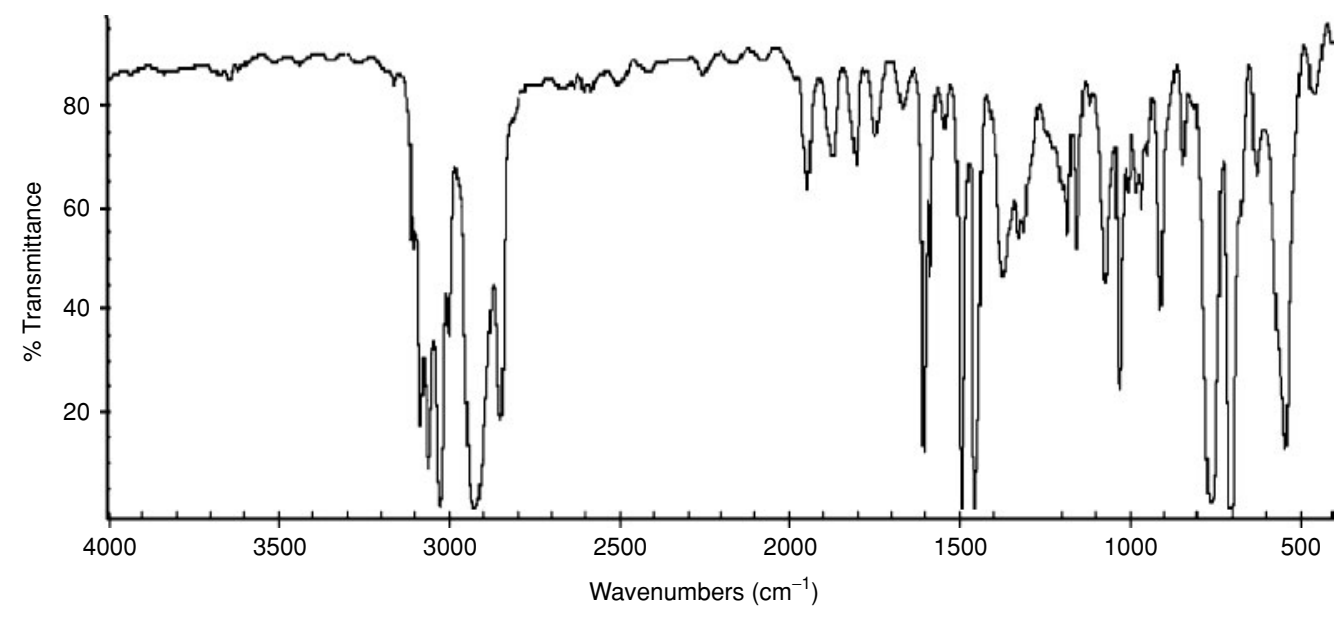

Figure 7 Absorption spectrum of the normalised blood.

of the absorption spectrum, and it is shown in Figure 7; in others words, taking the inverse of the frequency and multiplying it by the light speed.

$$
\begin{aligned}
H_{N} & =\sum_{k=0}^{N-1} \mathrm{e}^{2 \pi i(n k) / N} h_{k} \\
c & =\lambda \cdot f
\end{aligned}
$$

Figure 7 shows the absorption of red globules $(685 \mathrm{~nm})$, as well as of white globules $(1450 \mathrm{~nm})$. The width of the absorption peaks quantitatively determines the amount of the globules present. The response of the rotational modes between $2800 \mathrm{~nm}$ and $3100 \mathrm{~nm}$ is minimum for the hydroxyl groups (O-H) ( Zhbankov et al. 2000).

Figure 6 shows the absorption response for different wavelengths from several elements. Between them, we can find inorganic components and organic tailing of proteins, thus showing the complex nature of blood fluid .

\section{CONCLUSIONS}

In this research, an optical system was developed for the determination of some blood properties. An absorption spectrum is obtained by Fast Fourier Transform analysis. The system operates in an automatic manner. By changing the light modulation, it is possible to scan the finger at different depths. A correlation of the theoretical and experimental results for the optical system was obtained. The results obtained for the complete system were satisfactory. The software for the storage of the database with respect to time for a subsequent Fourier analysis was developed. The complete system is capable of analysing the optical absorption as well as the thermal absorption. Furthermore, this method of blood analysis is noninvasive.

\section{ACKNOWLEDGMENTS}

The authors thank Dr Carlos Rubio from Engineering \& Industrial Development Center (CIDESI) for his technical assistance and the financial support of CONACYT is also gratefully acknowledged.

\section{REFERENCES}

Cysewska-Sobuslack A. 1994. One dimensional representation of light tissue interaction for application in noninvasive oximetry. Opt Eng, 36(4):1225-33.

Gotshal Y, Simhi R, Sela B-A, et al. 1997. Blood diagnostic using fiber optic evanescent wave spectroscopy and neural networks analysis. Sens Actuators, 42:157-61.

Jacques SL. 1993. Role of tissue optics and pulse duration on tissue effects during high power irradiation. $A p p l O p t$, 32(13):2447-54.

Javidi B, Horner JL. 1994. Optical pattern recognition for validation and security verification. Opt Eng, 33:1752-6.

Nitzan M, Babchenko A, Milston A, et al. 1996. Measurement of the variability of the skin blood volume using dynamic spectroscopy. Appl Surf Sci, 106:478-82.

Vo-Dinh T. 2003. Biomedical Photonics Handbook. New York: CRC Press.

Zhbankov RG, Firsov SP, Korolik EV, et al. 2000. Vibrational spectra and the structure of medical biopolymers. Molec Struct, 555:85-96. 

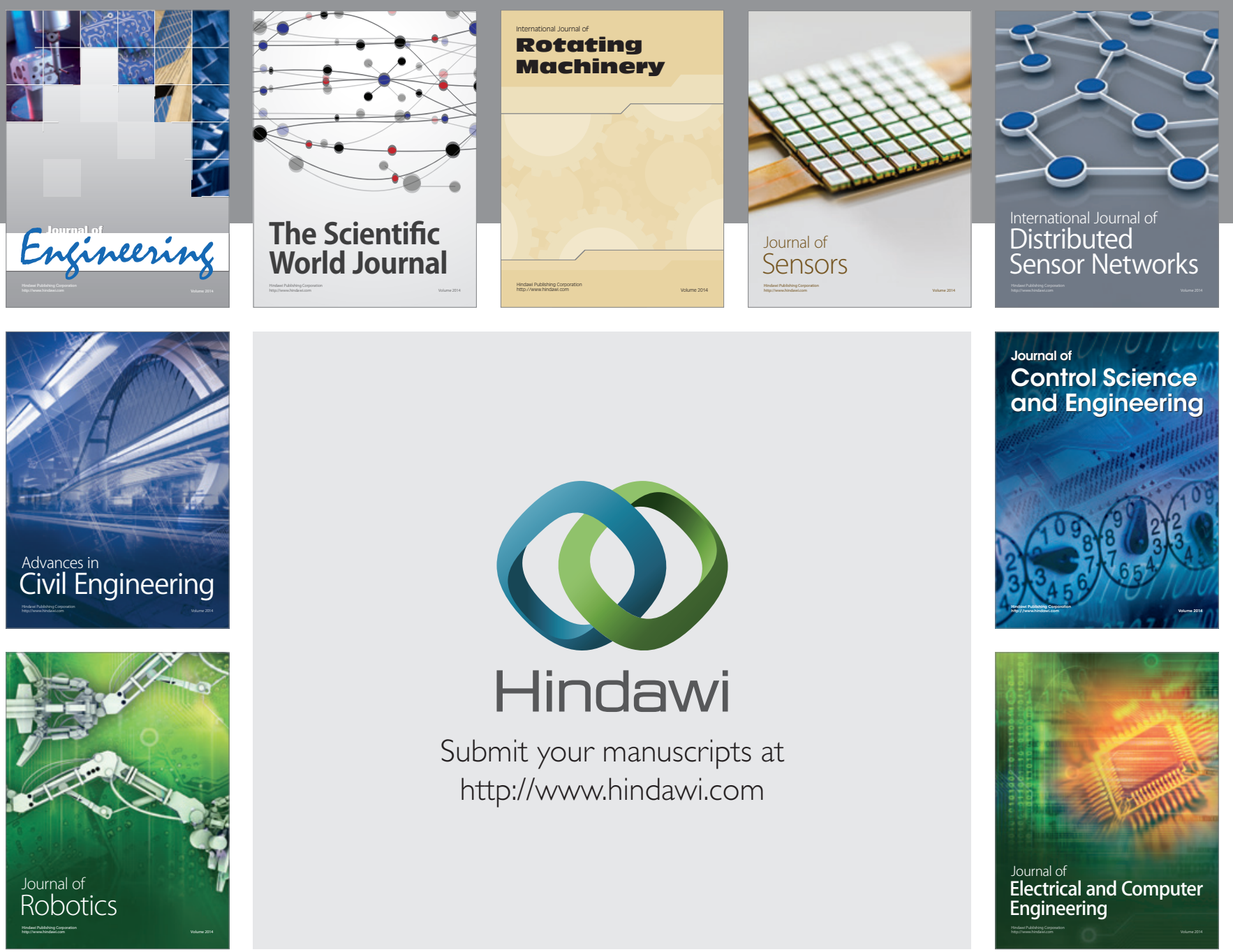

Submit your manuscripts at

http://www.hindawi.com
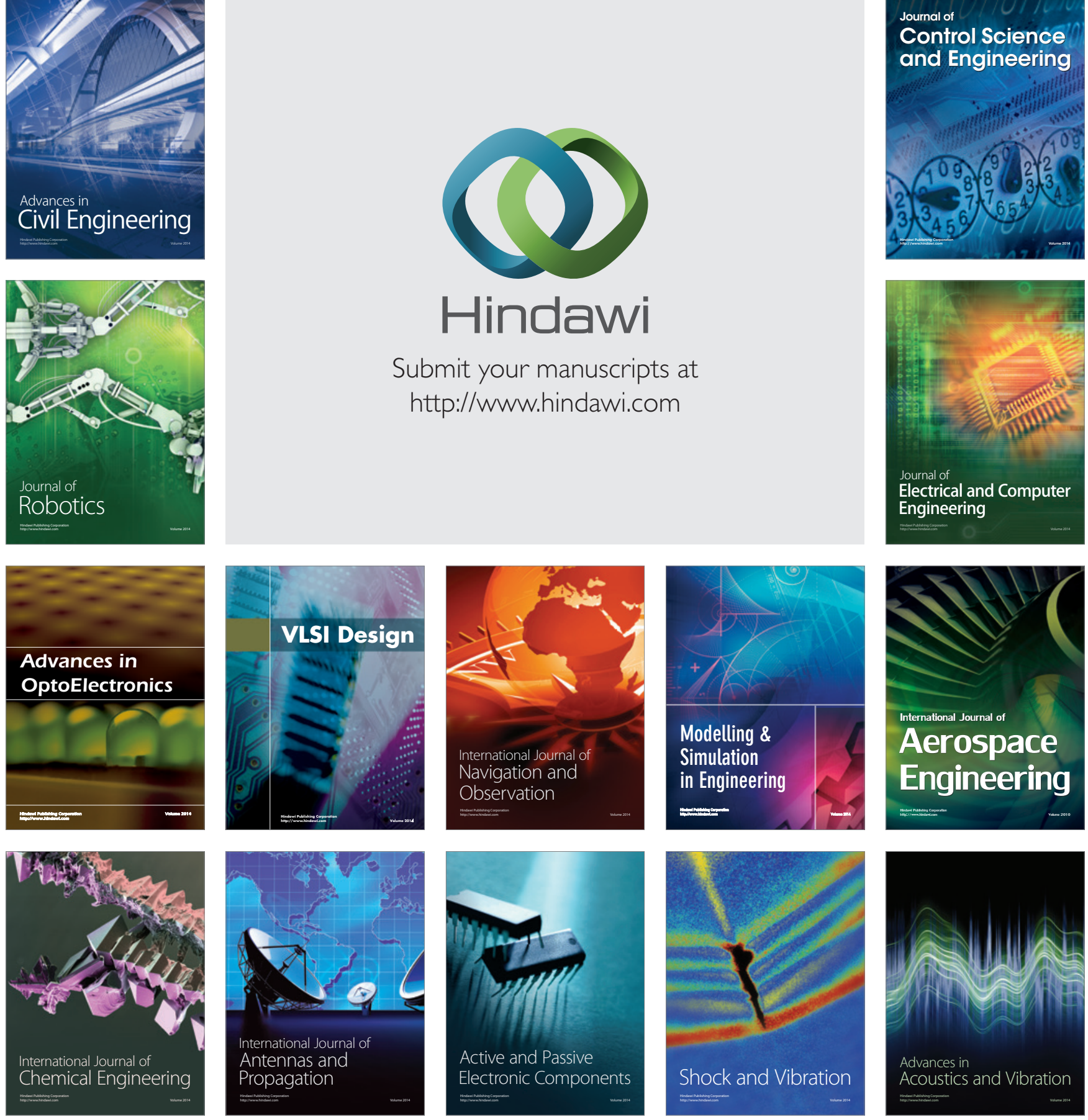